\title{
Mallarmé et la «valeur de bizarrerie » du poète comme ombre
}

\author{
Margot FAVARD \\ Université Paris Diderot
}

\begin{abstract}
L'obscurité est un des lieux communs majeurs entourant le nom Mallarmé, l'œuvre et le poète. Si elle lui est souvent reprochée comme preuve de son élitisme, Mallarmé en fait cependant le ressort de sa visibilité dans la communauté littéraire car elle lui confère une identité si particulière qu'elle en devient évidente et transmissible. C'est que l'obscurité a pour corollaire un autre lieu commun poétique, celui du lien privilégié de tout poète avec les ombres. Nous voulons ainsi interroger la manière dont Mallarmé s'attèle à une fabrique de l'ombre qui puisse répondre à la « crise de vers » scellée par la mort de Hugo, par une œuvre toute d'obscurité et une figure de poète nimbée dans l'ombre comme une apparition toujours disparaissant.
\end{abstract}

Keywords: Mallarmé, Hugo, obscurité, disparition, gloire

À vouloir aborder la question des valeurs du lieu commun avec Mallarmé, on est aisément tenté d'adjoindre l'obscurité au nom du poète et ainsi de reconduire ce qui est déjà un lieu commun critique. En 194I, Charles Mauron publie Mallarmé l'obscur' : le nom du poète reçoit son épithète homérique, et ce titre synthétise pour longtemps une des représentations les plus courantes de l'œuvre de Mallarmé, celle que bien des histoires littéraires, scolaires et universitaires reprennent, sans que cela ne signe pour autant son invalidité. C'est que le nom de Mallarmé, recouvrant homme et œuvre, paraît se prêter particulièrement à la création de clichés qui ont la peau dure.

Mallarmé spécialiste de la destruction des clichés de langue et pourfendeur des lieux communs du journal, voilà un autre lieu commun que notre étude voudrait un peu amender afin de considérer que ce poète-là puisse être aussi le poète qui produit du lieu commun. Ce changement de perspective implique de ne plus voir en Mallarmé la possible victime de clichés dont l'obscurité serait l'étendard, clichés produits par ceux qui le lisent en contre comme par ceux qui sont ses adeptes, et d'observer en quoi il peut être tenu pour responsable et créateur zélé des lieux communs qui entourent son nom d'auteur. C'est dans cette optique que j'interrogerai la fabrique de «Mallarmé l'obscur » par Mallarmé lui-même, soit la formation d'un cliché d'auteur par le premier concerné, qui en tire profit au risque calculé des pertes et

I Charles Mauron, Mallarmé l’obscur, Paris, José Corti, I94I. 
malentendus engendrés en chemin.

L'enjeu d'une possible efficace de son propre cliché est celui de la visibilité du poète dans le champ et l'histoire littéraire, son inscription dans une culture et une mémoire commune : son obscurité, à la fin du XIX ${ }^{\mathrm{e}}$ siècle, est déjà à la fois le lieu commun qui permet d'identifier le poète et la bizarrerie qui le sort du commun. Cependant, avant de devenir la preuve de son étrange singularité, l'obscurité de Mallarmé paraît être tributaire de manière essentielle d'un autre lieu commun poétique et mythique, qui n'a rien de spécifique à Mallarmé : celui du lien privilégié des poètes avec l'ombre et les ombres. Comment Mallarmé réinvestit-il ce lieu commun du poète en dialogue avec l'ombre pour y loger sa bizarrerie propre ? Comment réussit-il à se faire lui-même ombre, image projetée en noir, fiction de présence d'un sujet poétique pourtant présent?

\section{D'une « vieille image » qui circule dans deux lettres}

Dans le moment fondamental de sa « crise de Tournon » où se décide son esthétique, sa poétique et sa figure d'auteur, l'on voit déjà apparaître la lucidité de Mallarmé quant à l'usage bénéfique du lieu commun d'auteur. De I864 à I866 environ, alors qu'il s'essaie à poursuivre l'écriture d'Hérodiade, figure qui s'effraie de se voir dans son miroir « comme une ombre lointaine " Mallarmé éprouve un profond désespoir lié à sa stérilité poétique. Le poète n'est alors obscur que parce qu'il est un jeune poète peu connu. C'est dans les bornes initiales et finales de cette crise que Mallarmé joint une même photographie de lui dans deux lettres distinctes : la première est adressée à Frédéric Mistral le 30 décembre I864, la seconde est destinée à José-Maria de Heredia le 30 décembre i865. Il est difficile de savoir exactement quelle est cette photographie, mais il s'agit sans doute d'un des deux portraits du poète pris par Constantin en $\mathrm{I}^{86 \mathrm{I}^{3}}$. Mallarmé diffuse ainsi un portrait de lui à dix-neuf ans, à chaque fois dans une lettre de vœux de nouvel an, une des spécialités de Mallarmé, et à chaque fois le présent de la photographie est suivi, en post-scriptum, d'une sorte de courte légende :

J'ai là une vieille image : je vous l'envoie parce que le jour où je ne serai plus que mon ombre, et ce jour vient, elle aura une certaine valeur de bizarrerie ${ }^{4}$.

\footnotetext{
2 Stéphane Mallarmé, Hérodiade. Scène [1871, I899], in Euvres complètes, 2 vol., éd. Bertrand Marchal, Paris, Gallimard, «Bibliothèque de la Pléiade », I998-2003, t. I, p. 19.

3 Voir Jean-Michel Nectoux, Hervé Joubeaux et Jean-David Jumeau-Lafond (dirs.), Portraits de Mallarmé de Manet à Picasso, Vulaines-sur-Seine, Musée départemental Stéphane Mallarmé, 2013.

4 Mallarmé, lettre à Frédéric Mistral, 30 décembre I864, in Correspondance, éds. Henri Mondor et Lloyd James Austin, II vol., Paris, Gallimard, I959-I985, t. I, pp. I48-I49.
} 
Je retrouve un ancien portrait du temps où je sombrais dans la mer du Spleen, j'ai l'air, n'est-ce pas, d'un naufragé qui se résigne. Le voulez-vous? Et vous, tâchez aussi d'en retrouver un'.

Malgré leurs différences, les deux envois ont pour enjeu commun de servir au jeune poète de médiation vers deux auteurs consacrés, le Maître des Félibres et un des Maîtres du Parnasse, dont il aspire à être reconnu littéralement grâce à son portrait, comme à défaut et en vue d'une reconnaissance plus littéraire à venir. Avant de leur adresser ces présents, Mallarmé n'a rencontré qu'une fois Mistral et Heredia et il se rappelle ainsi à leur bon souvenir avec une certaine forme d'aplomb, ou de culot. L'envoi engage un système de don et de contre-don, nettement visible dans le mot à Heredia : puisque Mallarmé a offert son portrait, à Heredia maintenant de lui rendre la pareille. Quant à la lettre pour Mistral, elle était écrite « du fond de l'Ennui », de la crise, et Mallarmé y formulait le souhait de « sortir de là ! " ${ }^{6}$ Le souhait à l'infinitif vaut pour injonction adressée à Mistral, chargé désormais de l'aider à s'extraire de son désespoir et de son obscurité d'inconnu. Le système du don/contre-don instaure un lien qui importe autant si ce n'est plus que le bien échangé : la diffusion de son portrait crée ce lien avec des maîtres susceptibles d'aider le jeune poète à entrer dans le champ littéraire.

Le bien échangé importe tout de même bien sûr ainsi que les différences entre les deux légendes du portrait qui construisent deux légendes du jeune inconnu. Dans sa lettre à Mistral, du haut de ses vingt-deux ans, Mallarmé se pose en voix d'outre-tombe dont le devenir ombre ne saurait tarder. Cette mort projetée est, de manière topique, donnée comme la condition nécessaire de sa gloire qui ne peut survenir qu'après, et donc grâce à, sa transformation en ombre. C'est là le grand lieu commun poétique qui fait de la gloire du poète le corollaire de sa mort sur le modèle du chant du cygne, de la mort du Tasse lors de sa consécration au Capitole, ce que Balzac nomme encore « le soleil des morts » dans La Recherche de l'absolu?. Et c'est d'ailleurs sous le titre du Soleil des morts que le mallarmiste Camille Mauclair publie bien plus tard un roman où Mallarmé, qui écrivait n'avoir qu'« incompétence, sur autre chose que l'absolu ${ }^{8}$, apparaît comme personnage de fiction sous le nom de Calixte Armel ${ }^{9}$.

Mallarmé s'inscrit dans ce grand lieu commun poétique pour mieux prévenir Mistral de son futur glorieux, ce qui est une autre forme d'audace. En sus d'une reconnaissance dans le temps présent, le cliché photogra-

5 Id., lettre à José Maria de Heredia, 30 décembre 1865, in Correspondance, op. cit., t. III, p. 377.

$6 I d$., lettre à Frédéric Mistral, 30 décembre I864, op. cit.

7 Honoré de Balzac, La Recherche de l'absolu [1834], Paris, Gallimard, 1976, p. I74.

8 Mallarmé, «Solennité », in Divagations, in CEuvres complètes, op. cit., t. II, p. 198.

9 Camille Mauclair, Le Soleil des morts, Paris, P. Ollendorff, I898. 
phique doit garantir une reconnaissance future. L'image est envoyée pour assurer la persistance du poète après sa mort, elle en est déjà une ombre, chargée de rappeler en mémoire, et d'inscrire dans une mémoire et une culture poétique commune. Il faut souligner également la lucidité du jeune Mallarmé quant à l'aptitude de l'image d'écrivain à œuvrer pour construire sa valeur. Être une ombre est une des conditions de la gloire, diffuser une image de soi en est sans doute une deuxième. Or si l'image aura une "valeur de bizarrerie ", c'est que pour l'instant elle en est dépourvue. En effet, c'est là le portrait du poète non distingué et perdu dans la foule des auteurs, c'est le portrait du poète avant qu'il ne sorte du commun. Ainsi, d'une part, le commun peut connaître un « devenir bizarre », et, d'autre part, la bizarrerie peut permettre de sortir du commun, et pour cela peut-être n'est-il pas besoin d'être mort...

Enfin, l'image n'est bizarre que lorsqu'on la regarde du haut de la gloire de Mallarmé pour jouer à un curieux avant-après de la gloire. L'image provoque l'étonnement, rétrospectivement : dans ce portrait de Mallarmé, il n'est pas certain qu'on reconnaisse " Mallarmé » le poète célèbre, et pour cause il ne le deviendra que vingt ans plus tard, à quarante-deux ans, et il ne ressemble alors plus du tout à ce portrait.

À quoi ressemble-t-il d'ailleurs dans cette photographie ? Mallarmé répond lui-même dans sa lettre à Heredia en prenant ses distances avec le portrait. La photographie n'a que trois ans, mais elle déjà une "vielle image ", « un ancien portrait » d'un temps révolu avec lequel il creuse la distance temporelle. Â la date de ce second envoi, la crise s'est apaisée et Mallarmé vient de passer le nouvel an avec Heredia chez Leconte de Lisle. Cette fois, lettre et portrait installent une connivence qui repose sur la reconnaissance d'un code culturel partagé entre poètes du Parnasse. À Heredia, il demande en effet de reconnaître ("j'ai l'air n'est-ce pas ») un autre lieu commun poétique : le Spleen, la mer, le naufrage, la résignation, en somme tous les éléments d'un voyage baudelairien qui tourne mal. Mallarmé s'inscrit dans un cliché préexistant et dans une filiation littéraire dont simultanément il se distancie avec ironie pour s'adresser au maître parnassien. Il produit une image ambiguë de soi comme romantique baudelairien décalé, à distance, ou comme poète qui relègue le spleen dans les oubliettes de l'histoire littéraire. Cette mise en scène de son arrivée dans le champ poétique a encore une forte dose de provocation, car Baudelaire reste un de ses Maîtres incontestés. Mais ici, par le partage d'un cliché reconnu, il s'agit d'œuvrer pour l'inscription de Mallarmé dans la communauté poétique. En manipulant les clichés, il réalise une opération de communication qui réussit d'ailleurs plutôt bien puisqu'en I866 ses premiers poèmes paraissent dans Le Premier Parnasse contemporain.

Le jeune Mallarmé, l'obscur d'avant la gloire, s'inscrit ainsi dans le cli- 
ché du poète au devenir ombre et à distance du cliché du poète désespéré. On tient là la formule du positionnement et scénario auctorial de Mallarmé au sortir de la crise de Tournon : un devenir-ombre non désespéré, ou plutôt un déjà-ombre non désespéré. Dans une lettre à Henri Cazalis de janvier I865, le poète exprimait ce regret :

Être un vieillard, fini, à vingt-trois ans, alors que tous ceux qu'on aime vivent dans la lumière et les fleurs, à l'âge des chefs-d'œuvre ! Et n'avoir pas même la ressource d'une mort qui aurait pu faire croire, à vous, que j'étais quelque chose et que, si rien ne reste de moi, le Destin seul qui m'eût emporté doit être accusé ${ }^{10}$ !

C'est une modulation importante du lien entre gloire et mort qui fait de la mort non seulement la condition de la gloire mais surtout sa condition de légitimité : la mort a le pouvoir de « faire croire » à la gloire légitime d'un poète qui serait « quelque chose », et non « rien ».

Se décide alors un nouveau coup de dé mallarméen : la crise devient une traversée des Ténèbres dont le poète ressort ressuscité ${ }^{\prime \prime}$. En mai I867, il décrit à Cazalis sa longue agonie puis lui annonce aller bien mieux par cette étonnante formule : « heureusement je suis parfaitement mort $»^{12}$. Heureuse mort avant l'heure, parce qu'elle fait du poète une présence désormais marquée par l'absence et qui peut donc «faire croire » à son autorité et légitimité. Se déclarer " parfaitement mort », c'est pouvoir hâter son devenir ombre, en faire son état présent et acquérir dès maintenant une valeur. Deux ans plus tard, il réécrit cette résurrection : « J'ai imploré la grande Nuit, qui m’a exaucé et a étendu ses ténèbres. La première phase de ma vie a été finie. La conscience, excédée d'ombres, se réveille, lentement formant un homme nouveau [...] »'. Désormais cette « conscience, excédée d'ombres » déploie une poétique elle aussi « excédée d'ombres ", une poétique qui fait un emploi de l'ombre qui dépasse toute mesure. C'est par l'excès que Mallarmé réinvestit le cliché du poète comme ombre pour y loger sa singularité.

Io Mallarmé, lettre à Henri Cazalis, 30 mars ou 6 avril I865, in Correspondance, op. cit., t. I, p. 150.

II Voir la lettre à Frédéric Mistral, 3I décembre I865, ibid., p. I90 : " Le spleen m’a presque déserté [...]. L'impuissance est vaincue [...]. Merci de votre amicale prophétie, d'elle est née, sans doute, cette Résurrection ».

I2 Id., lettre à Henri Cazalis, I4 mai I867, ibid., p. 713.

I3 Id., lettre à Henri Cazalis, I8 ou I9 février I869, ibid., p. 30 I. 


\section{«- Es-tu mort ? - Presque. J'habite l'ombre " ${ }^{14}$}

Bien après " la crise de Tournon ", Mallarmé dans son poème critique "Crise de vers ", dans le morceau qui en paraît en I886, rend hommage à Victor Hugo comme «monument $»^{15}$ du siècle. Grand poète de l'ombre au XIX ${ }^{e}$ siècle, Hugo est aussi celui dont l'ombre plane sur le champ littéraire car dans la " crise de vers » se joue une crise du trône poétique, laissé vacant par la mort de Hugo. Mallarmé proclame ouvert l'interrègne, mais il en occupe néanmoins la régence. Il faut dire que par un étrange hasard, le poète accède à la reconnaissance l'année même où Hugo disparaît. Alors que «Crise de vers » notait que Hugo avait « confisqu[é] $»^{16}$ à tous la parole poétique, il semble que Mallarmé lui-même se soit appliqué en partie à «confisquer »à Hugo son ombre, ou du moins son lexique de l'ombre.

Nuit, ténèbres, obscurité, le lexique topique du poète qui écrit Les Rayons et les Ombres (I840) irrigue les écrits de Mallarmé et leur réputation d'obscurité tient aussi à cette présence littérale et récurrente des termes de l'ombre. Quand dans Les Feuilles d'automne le poète hugolien s'exclame avoir cru être une " vaine ombre obscure et taciturne, / Le roi mystérieux de la pompe nocturne $»^{17}$, Mallarmé paraît avoir pris cette identité au pied de la lettre. Il n'est pas celui qui écoute «ce que dit la bouche d'ombre $\aleph^{18}$, il est lui-même cette bouche d'ombre, ce spectre et « roi mystérieux ». Non content de s'approprier ce lexique de l'ombre par une sorte de braconnage poétique sur les terres de Hugo, il pousse la logique plus loin en créant une syntaxe de l'ombre. Pointe extrême du romantisme, Mallarmé radicalise l'ombre hugolienne pour en faire une bizarrerie qui lui soit propre. L'ombre règne à ce point chez Mallarmé qu'elle est au fondement de sa poétique dont l'enjeu est d'« évoquer, dans une ombre exprès, l'objet tu, par des mots allusifs, jamais directs $»^{19}$. Lorsque le téméraire Alphonse Daudet ose lui demander «si c'est volontairement [qu'il s'est] retiré dans des ténèbres » ou « involontairement », Mallarmé répond : « Mais est-ce que l'opération même d'écrire n'est pas de mettre du noir sur du blanc ? $»^{20}$. En s'appliquant à « mettre du noir sur du blanc », à propager l'ombre à tous les niveaux de sa poétique, Mallarmé fait de l'obscurité le lieu topique où s'unit son œuvre, et ce par quoi on peut aussi assez justement en synthétiser les enjeux poétiques. Elle offre une

14 Victor Hugo, «Écrit en I855 », in Les Contemplations [1856], Paris, Gallimard, I973, p. 253.

I5 Mallarmé, « Crise de vers », in Divagations, op. cit., t. II, p. 205.

I6 Ibid.

I7 Victor Hugo, « Parfois, lorsque tout dort... ", in Feuilles d'automne [183I], Euvres poétiques, 3 vol., éd. Pierre Albouy, Paris, Gallimard, «Bibliothèque de la Pléiade », I964-I974, t. I, p. 760. I8 Id., "Ce que dit la bouche d'ombre », in Les Contemplations, op. cit., p. 386.

19 Mallarmé, "Magie », in Divagations, op. cit., p. 25I.

20 Ce dialogue est rapporté par Georges Rodenbach dans son article "Stéphane Mallarmé ", Le Figaro, I3 septembre 1898. 
prise sur son œuvre qui est transmissible et intelligible.

\section{«À tous clarté, à moi ténèbres " ${ }^{2 \mathrm{I}}-$ Velut umbra}

Devenu «bouche d'ombre », le poète gagne une visibilité au ressort paradoxal car le système de l'obscurité frappe comme une forte anomalie dans le temps de l'essor des journaux et de l'alphabétisation. En extrayant celui qui la pratique hors de la langue commune, elle permet pourtant de contrer le risque de se trouver perdu parmi les « étalages $»^{22}$ surpeuplés des librairies. L'obscurité a cet avantage d'offrir une garantie contre l'invisibilité littéraire au temps de la démocratie et d'une égalité, annoncée, des discours et des individus.

Cette singulière bizarrerie qui le rend visible dans le champ littéraire dirige cependant vers lui le feu des critiques et des accusations d'élitisme, quand l'obscurité mallarméenne se voudrait une obscurité valable pour tous. Le règne de l'ombre chez Mallarmé devient la preuve de sa mystification et de sa fumisterie poétique, et pourquoi pas de sa magie noire. Gustave Lanson, présentant en juillet I893 « la poésie contemporaine », voit en lui :

comme une puissance obscure et lointaine [...], une sorte de Vieux de la Montagne invisible et inquiétant, qui lâchait à travers la littérature des émissaires fantastiques, grisés de merveilleuses visions et propagateurs d'incompréhensibles mots d'ordre ${ }^{23}$.

Rien de ces attaques n'échappe à Mallarmé et il y répond dans « Le Mystère dans les lettres » en se déclarant « suppôt d'Ombre, d'eux désigné »². Si ce « mystère » doit être préservé, c'est aussi parce qu'attisant les critiques, il offre à ces écrits la visibilité inhérente aux points de débats du champ littéraire.

Mais le « suppôt d'Ombre » est tout aussi bien un Dieu de l'Ombre pour ses admirateurs qui se rassemblent autour du poète en une communauté protectrice : le «Vieux de la montagne » est entouré de son armée des ombres qui se charge de diffuser sa gloire. Dans leurs éloges, les mallarmistes font d'ailleurs de son obscurité le ressort de sa grandeur, et inversement. Charles Mauron le résume ainsi : «L'obscurité du poète n'est pas plus niable que sa grandeur [...]. [U]n grand poète obscur, monstre jadis inimaginable, tout à

2I Maurice Scève, « La Chandelle et le Soleil » (6e emblème), in Délie [1544], Paris, Nizet, 1987, p. 42.

22 Voir Mallarmé, «Étalages », in Divagations, op. cit., pp. 218-223.

23 Gustave Lanson, "La Poésie contemporaine - M. Stéphane Mallarmé », La Revue universitaire, 7 , I5 juillet 1893 , p. 122.

24 Mallarmé, «Le Mystère dans les lettres », in Divagations, op. cit., p. 230. 
coup surgi $[t]$, grand sans conteste, obscur sans conteste, en pleine littérature française $»^{25}$.

Le «monstre » Mallarmé, c'est le poète dont la singularité frappe presque immédiatement parce que l'obscurité est patente. Et cette bizarrerie qui n'a rien de commun lui assure une forme d'autorité poétique. Le poète veille alors à la diffuser et à ne pas dissiper ce lieu commun qui pourrait jouer contre lui. C'est la célèbre conclusion de "Solitude » : le poète se met en scène face à un journaliste et le retient afin d'« ajoute[r], du moins, un peu d'obscurité $»^{26}$ à ses propos. Le poème critique tient à distance ironique la presse, le rite de la visite au grand écrivain tout en manifestant un emploi délibéré de l'obscurité. Le poète donne sa rieuse bénédiction à la fabrique de lieu commun le concernant et à sa diffusion. Le poète est inintelligible, peut-être, mais il est poète visible et reconnu en tant qu'obscur.

L'autre avantage que retire Mallarmé à se faire bouche d'ombre est la sacralité qu'elle lui procure. L'épigraphe de la «Préface testamentaire » des Mémoires d'outre-tombe de Chateaubriand est « Sicut nubes... quasi naves... velut umbra (Job) $»^{27}$, et si l'on en croit aussi l'avant-propos de ces Mémoires, être « comme une ombre ", voilà qui permet de s'approcher « de ces voix qui ont quelque chose de sacré, parce qu'elles sortent du sépulcre ${ }^{28}$. Voix sacrées parce que voix d'une résurrection, les voix de l'ombre sont aussi sacrées parce qu'elles projettent une ombre d'autorité sur ceux qui la détiennent. Pascal Durand et Patrick Thériault ${ }^{29}$ ont démontré la portée oraculaire de l'énoncé mallarméen : le discours de l'ombre est un discours de Sybille dont le mystère autorise de nombreuses interprétations en même temps qu'elle ouvre à un sentiment religieux. Ainsi fait-on croire qu'il y a quelque chose, au risque de découvrir qu'il n'y a rien, ce qui est toujours quelque chose. On peut y trouver un écho dans le Livre, majuscule, que Mallarmé annonce sans cesse à ses disciples mais ne finit jamais : le Livre devient pure ombre projetée d'un objet jamais réalisé, absent. À quoi peut faire encore écho ce mot laissé juste avant sa mort à propos de notes qu'il demande de brûler : «Croyez que ce devait être très beau " ${ }^{30}$.

25 Mauron, Mallarmé l'obscur, op. cit., p. I.

26 Mallarmé, «Solitude », in Divagations, op. cit., p. 259.

27 François-René de Chateaubriand, "Préface testamentaire " [1833], in Mémoires d'outretombe [1849-I850], 6 vol., Paris, Garnier, I9IO, t. I, p. XLIII.

28 Id., "Avant-propos" [1846], in Mémoires d'outre-tombe, op. cit., t. I, p. LIII.

29 Voir Pascal Durand, Mallarmé. Du sens des formes au sens des formalités, Paris, Seuil, 2008; et Patrick Thériault, «Le 89, rue de Rome. À l'enseigne du secret », Romantisme, 2012, pp. 698I ; ainsi que Le (Dé)montage impie de la fiction. La révélation moderne de Mallarmé, Paris, Honoré Champion, 2010.

30 Mallarmé, lettre à Marie et Geneviève Mallarmé, 8 septembre I898, in Euvres complètes, t. I, p. 82I. 


\section{"Cet examen des ténèbres. C'est l'étude d'un effacement " ${ }^{31}$}

Dans "Toast funèbre ", sonnet écrit pour le tombeau collectif de Théophile Gautier en I873, Mallarmé avertissait : " Ô vous tous! oubliez une croyance sombre. / Le splendide génie éternel n'a pas d'ombre ${ }^{32}$. La «croyance sombre » qu'il convient d'oublier est celle de la promesse de la résurrection catholique, d'un retour du poète comme ombre, à laquelle Mallarmé substitue la croyance lumineuse que la seule ombre qui existe est celle des œuvres. Mais puisque lui-même n'est pas au tombeau et que l'époque est à la porosité entre l'homme et l'œuvre, il poursuit son œuvre au noir et son travail de l'ombre dans sa vie littéraire, dans et hors de ses œuvres.

L'emprise de l'ombre chez Hugo était liée à l'exil du poète, et dans ses Contemplations s'élevait cette inquiétude : «Aujourd'hui dans une île [...], on ne me voit plus, tant je suis couvert d'ombre $»^{33}$. Hugo tire cependant profit de l'invisibilité offerte par l'île car l'exil fait du poète absent l'ombre qui plane sur la France, et c'est sur ce « on ne me voit plus » que se reconfigure sa visibilité et son autorité poétique. Ce qui n'est pas sans poser problème pour notre étude du réinvestissement par Mallarmé de l'ombre hugolienne. Car Mallarmé ne dispose pas plus de l'exil qu'il n'a de figure politique majeure avec et contre quoi construire son autorité. Alors que le devenir ombre de Hugo trouve une cause et une raison par l'exil, pour Mallarmé une telle logique ne tient plus. Comment être une ombre alors qu'on n'est ni mort ni exilé ? Mallarmé s'engage dans cette étrange entreprise de se rendre visible comme ombre et de rendre visible son effacement alors qu'il est là.

Voici quelques mots rassemblés du premier roman que Hugo écrit en exil, Les Travailleurs de la mer, et de son chapitre «Sub umbra»:

L'obscurité nocturne est pleine d'un vertige. Qui l'approfondit s'y submerge et s'y débat. Pas de fatigue comparable à cet examen des ténèbres. C'est l'étude d'un effacement. / Aucun lieu définitif où poser l'esprit. Des points de départ sans point d'arrivée. L'entrecroisement de solutions contradictoires, tous les embranchements du doute s'offrant en même temps, la ramification des phénomènes [...] l'invisible devenu vision. C'est l'Ombre. L'homme est là-dessous. / [...] On ne peut rien saisir. On est écrasé par l'impalpable. / Partout l'incompréhensible ; nulle part l'inintelligible. / [...] On est sous l'ombre. On regarde. On écoute. / [...] Quelle proposition de toutes les énigmes à la fois ! / L'irréductible est là. / On est contraint à la foi. Croire de force, tel est le résultat ${ }^{34}$.

3I Victor Hugo, Les Travailleurs de la mer [I866], Paris, Flammarion, 20I2, p. 426.

32 Mallarmé, «Toast funèbre ", in Poésies, in Euvres complètes, op. cit., t. I, p. 28.

33 Hugo, "À Jules Janin ", in Les Contemplations, op. cit., p. $26 \mathrm{I}$.

34 Id., Les Travailleurs de la mer [1866], op. cit., pp. 426-428. 
On serait presque tenté de lire dans ces lignes un écho de la poétique de Mallarmé : art des contradictions, du suspens et de l'instabilité du sens, des reflets entrecroisés des mots. Surtout, il me semble que chez Mallarmé «l'examen des ténèbres » engage une « étude » et un travail de l'effacement du poète : il s'essaye à trouver la formule d'une absence qui se fasse voir et qui soit fondée sur une présence pour mieux créer les conditions d'un croire poétique. C'est aussi que l'ombre hugolienne et l'effacement mallarméen sont un dialogue engagé par les deux poètes avec les ombres enfantines de Léopoldine et d'Anatole, qui posent de manière particulièrement aiguë la question des "fiction[s] de l'absence ${ }^{35}$, mais aussi celle des fictions de présence.

\section{« Fiction de l'absence », fictions de présence mallarméennes}

J'évoquerai trois de ces possibles fictions de l'absence-présence par lesquelles Mallarmé se manifeste comme présence et comme ombre dans un dédoublement qui les trouble toutes deux. On trouve l'esquisse de la première dans la lettre à Cazalis de mai 1867, où le poète se disait «parfaitement mort » : ayant constaté son " horrible sensibilité », le temps était désormais venu de «l'enveloppe[r] d'une indifférence extérieure ». Et il ajoutait encore : «C'est t'apprendre que je suis maintenant impersonnel et non plus

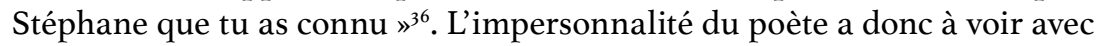
la double décision d'être déjà une ombre et de ne manifester qu'une "indifférence extérieure ». Celui qui prône la « disparition élocutoire » n'en écrit pas moins quantité de lettres qui manifestent sa présence auprès de ses contemporains. Dans les lettres où il accuse réception des ouvrages qu'on lui envoie, le paradoxe est que son «indifférence » se manifeste par une très grande bienveillance à l'égard de tous. Mallarmé, et non plus Stéphane, est un poète extrêmement poli ou d'une politesse si extrême avec tous $\mathrm{s}^{37}$ qu'elle lui fait atteindre une neutralité de jugement. C'est ainsi que la très grande présence épistolaire du poète produit un effacement de présence, l'effacement d'un jugement critique qu'on pourrait attendre d'un Maître. La subjectivité du poète se voile derrière l'excès de politesse qui le lie à tous en même temps qu'elle le maintient à distance de tous.

Une autre « fiction de l'absence » du poète s'esquisse lorsqu'il présente ses séjours dans sa villégiature comme autant de petits exils volontaires et

35 Mallarmé, « Notes pour un Tombeau d'Anatole », feuillet I86, in Euvres complètes, op. cit., t. I, p. 939.

36 Id., lettre à Henri Cazalis, I4 mai I867, op. cit.

37 Ce qui conduira Jean-Paul Sartre à déclarer que le poète « choisit le terrorisme de la politesse ", dans son article de 1953, repris dans Mallarmé. La lucidité et sa face d'ombre, Paris, Gallimard, I986, p. I5I. 
autant de « disparition[s] élocutoire[s] $»^{38}$. Au bord de la Seine, la petite maison de Valvins est un Guernesey miniature, où le poète disparaît rituellement et temporairement. À chaque départ, se joue la fiction d'une absence définitive qui est dûment signalée à ses amis : il y a « disparition élocutoire ", parce que le poète dit qu'il va disparaître. Mais à Valvins, Mallarmé continue à envoyer quantité de lettres, planant ainsi comme ombre à Paris, et veille à assurer de son retour prochain. Il exhibe ses disparitions et ses réapparitions, son invisibilité et sa visibilité dans une alternance qui définit le rythme de sa maîtrise : il est une autorité et une présence toujours disparaissante, et qui d'ailleurs finira par disparaître tout à fait là-bas.

Enfin, une troisième «fiction de l'absence » se joue dans le lieu même où elle paraît impossible puisqu'il s'agit de son salon de la rue de Rome où il réunit son cénacle le Mardi, là où sa présence est incontestable. Alors qu'il apparaît devant les mardistes, son public assis devant lui comme au théâtre, comment Mallarmé peut-il se montrer aussi comme ombre, fiction de présence? Il y parvient notamment par le dispositif scénique et optique qui met à ses côtés deux tableaux de Manet. L'un est son portrait peint en I876 qui est, selon Mallarmé, un « curieux tableautin qui le représente à une époque déjà ancienne " $^{39}-$ une "vieille image » donc, à nouveau. La reproduction du tableau accompagne la parution des Poètes maudits de Verlaine qui y voit un Mallarmé « en quelque sorte apothéosé, immortalisé »40. Ainsi, devant les yeux des mardistes surgit un double Mallarmé ou les deux corps du Prince des poètes : un Mallarmé présent et causant, leur Maître, et un Mallarmé représenté, modèle de Manet à l'époque ancienne de son relatif anonymat. À cela, il faut ajouter un pastel de Manet, peint en I877 et offert à Mallarmé en I883, où Hamlet se débat contre le spectre, une silhouette si peu esquissée dans le pastel qu'il se bat contre une silhouette blanche, contre un vide. Le dispositif des Mardis permettait donc sans doute d'observer, à côté de Mallarmé en chair et en os, à la fois l'image de sa jeunesse et l'image d'Hamlet, double analogique d'un poète qui combat le Néant. Ainsi entouré, le poète pourtant présent est tout près d'entrer lui-même dans la fiction, d'être déjà un « Mallarmé imaginaire $»^{4}$.

Camille Mauclair, dans ses souvenirs de Mallarmé chez lui, déplorait que les mardistes l'aient " embaumé vivant ${ }^{42}$. Il n'est pas certain que Mallarmé partage ce regret, bien au contraire, car l'embaumement vivant est une

38 Mallarmé, «Crise de Vers », in Divagations, op. cit., p. 2 II.

39 Id., lettre à Verlaine, 22 août I883, in Correspondance, op. cit., t. II, p. 245.

40 Paul Verlaine, "Avertissement à propos des portraits ci-joints ", in Les Poètes Maudits [I884], Euvres en prose complètes, éd. Jacques Borel, Paris, Gallimard, "Bibliothèque de la Pléiade », 1972, p. 636.

4I Henri de Régnier, "Les Portraits de Mallarmé ", in Nos rencontres, Paris, Mercure de France, 193I, p. I84.

42 Camille Mauclair, Mallarmé chez lui, Paris, Grasset, 1935, p. 62. 
forme de solution, aussi bizarre soit-elle, pour être une ombre vivante, une fiction de présence. C'est ce qui surgit peut-être dans la célèbre photographie que Degas prend de Mallarmé et de Renoir en 1895 : le photographe disparaît dans le miroir et la lumière, tandis que le poète, yeux baissés ou fermés, paraît s'absenter. L'ombre présente, c'est peut-être celle enfin que seul Whistler réussit à représenter en 1892 dans une lithographie où, selon Georges Rodenbach, « le poète [est] déjà en un recul, hors du temps, tel qu'il apparaîtra à l'avenir ${ }^{43}$. " Ce portrait est une merveille, Mallarmé remercie-t-il Whistler, la seule chose qui ait jamais été faite d'après moi, et je m'y souris $»^{44}$ : merveille en effet d'un portrait qui n'a rien d'obscur, où le poète est tout ombre; le portrait d'un effacement.

Sous l'ombre protectrice de l'obscurité, Mallarmé règne " par l'étonnant pouvoir de l'absence ${ }^{45}$ sur le siècle qui lui survit, par l'étonnant pouvoir de sa présence éditoriale aussi, assurée par la publication posthume de nombreuses œuvres. Un lieu commun en cachant souvent un autre, « Mallarmé l'obscur » se métamorphose bientôt en « Mallarmé le blanc » : le xx siècle est hanté par l'ombre des écrits de celui en qui elle voit le poète de l'effacement, de l'impersonnalité, de la vacance, et le responsable de la mort de l'auteur, nouveaux lieux communs dont la modernité fera profit, nouvelle gloire du poète.

43 Rodenbach, «Stéphane Mallarmé », art. cit.

44 Mallarmé, lettre à Whistler, 5 novembre I892, in Correspondance, op. cit., t. V, pp. I43-I44.

45 Victor Segalen, «Éloge et pouvoir de l'absence », in Stèles [1912], Paris, Mercure de France, I982, p. 25 I. 